\title{
Angular Momentum Minimal Magnetization of an Elementary Quantum Fermion System
}

\author{
C. Verzegnassi \\ Dipartimento di Fisica, Università di Trieste, INFN Sezione di Trieste, Italy \\ Email: claudio@ts.infn.it
}

Received February 26, 2013; revised March 27, 2013; accepted April 24, 2013

Copyright (C) 2013 C. Verzegnassi. This is an open access article distributed under the Creative Commons Attribution License, which permits unrestricted use, distribution, and reproduction in any medium, provided the original work is properly cited.

\begin{abstract}
I consider, in a Quantum Field Theory theoretical approach, the effects of an electromagnetic field on the components of the total angular momentum of an elementary fermion system, assuming the "minimal" form of the relative interaction. When the electromagnetic field can be treated as a classical one, these effects are particularly simple to be computed and exhibit a number of very general characteristic features in the case of a constant magnetic field. A qualitative possible analogy with similar features of an elementary organic system is finally proposed.
\end{abstract}

Keywords: Organic

\section{Introduction}

The motivation of this paper comes from a very recent medical Conference held in Trieste on February 15th, studying the relationship between stem cells and cancer [1], and from several explanations that I received from the two main speakers of the Conference, M. Biava and F. Burigana. In particular it was stressed that the Conference organizing association, AMEC [2], is at the moment supporting studies to activate the stem cells with electromagnetic fields. Previously, I had already found on a book [3] an article by P. Girdinio [4] with title: "An introduction to electromagnetic fields and their biological effects". Without entering the medical details of the paper, that are beyond my very limited knowledge, I will quote the two following sentences:

1. "In recent years a widespread discussion about the possibility of negative effects of electromagnetic fields on human health has been going on."

2. "All authors agree on the fact that the magnetic component of the field alone is responsible of health effects. This is due to the fact that the electric component of the field is strongly diminished by the shielding effect of natural and artificial obstacles [...]"

More recently, I also found the following statements of C. Ventura $[5,6]$ :

1. "Embryonic stem cells of mice. under the action of a low frequency magnetic field, are differentiated [...]"

2. "Embryonic stem cells of mice have been exposed to a new medical instrument, REAC, which has been built to utilize the beneficial effects of electromagnetic fields on the human body."

My conclusion is that it appears in medicine that a magnetic field alone will have effects, either positive or negative, on the elementary components of the known existing human organism.

The aim of this paper is to show that a similar situation appears in physics, i.e. that a magnetic field alone has effects on the elementary components of the known existing stable matter. In particular, I will concentrate on an elementary one fermion state, chosen to be the state of one electron. The treatment that I will give will be based on the relativistic quantum field theory description, and all my notations will follow those of a book written by M. Peskin and E. Schroeder [7]. In this theoretical framework, I will show that there exists one physical property of the electron, i.e. its angular momentum (to be defined in the next Section), that will be modified in a predictable way by the presence of a magnetic field alone. The organisation of the paper will be the following: in the next Section 2, I will define the main features of the angular momentum for a free electron state. The specific interaction with a magnetic field ("minimal" interaction) will be discussed in Section 3, and its effects will be derived. In Section 4, a short and very personal proposal of a possible analogy between the modifications of an elementary matter component and those of an elementary organic component that would be produced by the same magnetic field will be exposed. 


\section{The Angular Momentum of a Free One Fermion System in Relativistic Quantum Field Theory}

The relevant starting quantity of the approach will be a conventional four-components spinor field. To simplify the notations, since spinors are known to obey FermiDirac statistics, I will call this field a fermion field, and define it as $\psi(x)$, where $\boldsymbol{x} \equiv\left(x^{0}, x^{1}, x^{2}, x^{3}\right)$ is the conventional controvariant space-time four-vector. In the quantum approach, the field is an operator. Following the Peskin-Schroeder notations, I will write for $\psi(x)$ and for its "complex" associated field $\bar{\psi}(x)$ the following Fourier expansions

$$
\begin{aligned}
& \psi(x) \\
& =\int_{-\infty}^{+\infty} \frac{\mathrm{d}^{3} p}{(2 \pi)^{3}} \frac{1}{\sqrt{2 E_{p}}} \sum_{s}\left(a_{p}^{s} u^{s}(p) \mathrm{e}^{-\mathrm{i} p \cdot x}+b_{p}^{s ?} v^{s}(p) \mathrm{e}^{p \cdot x}\right) \\
& \bar{\psi}(x) \\
& =\int_{-\infty}^{+\infty} \frac{\mathrm{d}^{3} p}{(2 \pi)^{3}} \frac{1}{\sqrt{2 E_{p}}} \sum_{s}\left(b_{p}^{s} \bar{v}^{s}(p) \mathrm{e}^{-\mathrm{i} p \cdot x}+a_{p}^{s ?} \bar{u}^{s}(p) \mathrm{e}^{p \cdot x}\right)
\end{aligned}
$$

Here $p \cdot x=p_{0} x^{0}+\sum_{i=1}^{3} p_{i} x^{i}, p_{0} \equiv E_{\boldsymbol{p}}=\sqrt{\boldsymbol{p}^{2}+m^{2}}$, where $m$ is the fermion mass. The quantities $a_{p}^{s}, a_{p}^{s \dagger}, b_{p}^{s}, b_{p}^{s \dagger}$ are operators with a precise meaning: $a^{\dagger}$ and $b^{\dagger}$ are creators, $a$ and $b$ are destructors. In particular, defining the vacuum ket $|0\rangle$ as the state of minimum energy, $a_{p}^{s}$ and $b_{p}^{s}$ applied to the vacuum annihilate it, $a^{s}|0\rangle=b^{s}|0\rangle=0$. On the contrary, in the chosen convention, $a_{p}^{s \dagger}$ and $b_{p}^{s \dagger}$ applied to the vacuum generate a one particle fermion $\left(a^{\dagger}\right)$ and antifermion $\left(b^{\dagger}\right)$ state of momentum $\boldsymbol{p}$, energy $E_{\boldsymbol{p}}$ and electric charge of opposite sign, that will be called $e$ for an electron state and $(-e)$ for a positron state, with the conventional choice of negative $e$. The index $s=1,2$ will be related to the spin component along some direction (e.g. the $\mathrm{z}$ axis for simplicity) of the state. The quantities $u(\boldsymbol{p}), v(\boldsymbol{p}), \bar{u}(\boldsymbol{p}), \bar{v}(\boldsymbol{p})$ are column and row 4-components functions of given four-momentum $\left(E_{\boldsymbol{p}}, \boldsymbol{p}\right)$, whose expression is given in ref. [7]. The field $\bar{\psi}(x)$ is equal to $\psi^{\dagger}(x) \gamma^{0}$, where $\gamma^{0}$ is one of the four

$\gamma^{\mu}=\left(\gamma^{0}, \gamma^{1}, \gamma^{2}, \gamma^{3}\right)$ matrices that are also listed in [7], $\psi^{\dagger}(x)$ is the row four-dimensional vector containing the complex conjugate components of the column vector $\psi(x)$, and I hope that this brief and essential exposition may be sufficient for the purposes of this paper.

The definition of the total angular momentum of a fermion field $\psi(x)$ is given by the fundamental Noether's theorem [8]. This defines the considered three components observable, denoted with a vector notation $\boldsymbol{J}=\left(J^{1}, J^{2}, J^{3}\right)$, as the set of those 3 "charges" that are conserved in time as a consequence of the "rotational invariance" of the system, i.e. of the fact that there exists a symmetry of the laws of motion under the three space rotations. Calling $J^{i}$ the conserved component of the total angular momentum associated with the $i$-th space rotations $R^{i}$ (with the convention that one can define $R^{1} \equiv R_{23}, R^{2} \equiv R_{31}$ and $\left.R^{3} \equiv R_{12}\right)$, one finds after conventional standard manipulations that. quite generally, each component of the total angular momentum of a free fermion system can be written as a sum of two essentially different terms, i.e.:

$$
\boldsymbol{J}=\boldsymbol{L}+\boldsymbol{S}
$$

where

$$
\boldsymbol{L}=\int_{-\infty}^{+\infty} \mathrm{d}^{3} x \psi^{\dagger}(x)[\boldsymbol{x} \wedge(-\mathrm{i} \nabla)] \psi(x)
$$

where $\nabla$ is the "derivative vector", $\nabla_{\mathrm{i}}=\partial / \partial x^{\mathrm{i}}, \wedge$ denotes the "vector product" and, using the standard notation $S^{\mathrm{i}}=\epsilon^{\mathrm{i} l m} S_{l m}(l, m=1,2,3), S_{l m}=-S_{m l}$ :

$$
S_{l m}=\frac{1}{2} \int \mathrm{d}^{3} x \psi^{\dagger}(x) \sigma_{l m} \psi(x)
$$

where $\sigma_{l m}=\mathrm{i} \gamma^{l} \gamma^{m}$. The two terms $\boldsymbol{L}$ and $\boldsymbol{S}$ are the conventional orbital angular momentum and spin angular momentum components of the total angular momentum of the fermion field. Note that for a fermion field it is only the sum of the two quantities that remain constant in time, while this is not necessarily always true for the two separate components. In order to fix the chosen convention, I will consider the mean values of the angular momentum components when the field system is a one particle fermion state of three-momentum $\boldsymbol{k}_{0}$ and "spin" index $s_{0}$. In the Peskin-Schroeder normalization this state, that I will call $\left|\boldsymbol{k}_{0}^{s_{0}}\right\rangle$, is defined as:

$$
\left|\boldsymbol{k}_{0}^{s_{0}}\right\rangle=\sqrt{2 E_{\boldsymbol{k}_{0}}} a_{\boldsymbol{k}_{0}}^{s_{0} \dagger}|0\rangle
$$

where $E_{\boldsymbol{k}_{0}}=\sqrt{\boldsymbol{k}_{0}^{2}+m^{2}}$. From the rules that fix $u^{s_{0}}\left(\boldsymbol{k}_{0}\right)$ and the $\gamma$ matrices, one obtains in a straightforward way the requested mean values. The definition of mean value implies the calculation of quantities of the kind

$$
\langle\boldsymbol{J}\rangle_{\boldsymbol{k}_{0}, s_{0}} \equiv \frac{\left\langle\boldsymbol{k}_{0}^{s_{0}}|\boldsymbol{J}| \boldsymbol{k}_{0}^{s_{0}}\right\rangle}{\left\langle\boldsymbol{k}_{0}^{s_{0}} \mid \boldsymbol{k}_{0}^{s_{0}}\right\rangle}
$$

where the (in principle, infinite) norm of the state appears in the denominator (to cancel an analogous term in the numerator) and the $\boldsymbol{J}$ operator in the numerator must be written as the conventional "normal product" of $\boldsymbol{J}$, to avoid the presence of fictitious infinities. For simplicity, I will fix the direction of $\boldsymbol{k}_{0}$, e.g. along the 
chosen $z$ axis, so that $\boldsymbol{k}_{0}=\left(0,0, k_{0 z}\right)$. After a number of simple calculations one then finds for the components of the spin of the state:

$$
\begin{aligned}
& \left\langle S_{12}\right\rangle_{\boldsymbol{k}_{0}, S_{0}} \equiv\left\langle S^{3}\right\rangle= \pm \frac{1}{2} \\
& \left\langle S_{23}\right\rangle=\left\langle S_{31}\right\rangle=0
\end{aligned}
$$

and in (8) the positive sign corresponds to $s_{0}=1$, the negative one to $s_{0}=2$. As one sees, the chosen normalization reproduces the standard results that fix the components of the spin for the chosen state.

In the case of the orbital momentum, a similar calculation produces the results

$$
\left\langle L^{3}\right\rangle_{\boldsymbol{k}_{0}}=0
$$

For the two remaining components, the mean values do not have a physical meaning for the chosen free state of fixed momentum. In practice, they are infinite, given the fact that the particle has no space limits. A realistic calculation should be done using an initial "wave-packet" state, but for the purposes of this paper it will be sufficient to consider a state of vanishing $\boldsymbol{k}_{0}$. Then, also the remaining components of the orbital momentum mean values vanish, i.e.:

$$
\left\langle L^{1}\right\rangle_{0}=\left\langle L^{2}\right\rangle_{0}=0
$$

Equations (8)-(11) provide the information that I will use, for the case of a free quantum one fermion state. Although they might describe the angular momentum of an arbitrary fermion (e.g. lepton or quark), I will consider them from now on for the specific case of a one electron system. In the next Section 3, I will consider the modifications of the derived angular momentum expressions that are produced by an interaction of the fermion system with an electromagnetic field.

\section{Interaction with an Electromagnetic Field}

In a quantum field theory approach, the interaction between a fermion field and an electromagnetic one is fixed by the "historical" prescription, derived from the analogy with the classical physics description of the interaction between a charged particle and a classical electromagnetic field. In the field theory description, the so called "minimal" interaction is obtained by replacing the usual derivative $\partial_{\mu}=\frac{\partial}{\partial x^{\mu}}$ by the "covariant" derivative

$$
D_{\mu}=\partial_{\mu}+\mathrm{i} e A_{\mu}(x)
$$

where $A_{\mu}$ is the electromagnetic vector potential and $e$ is the fermion charge. From the replacement (12) the expression of the electromagnetic interaction, and of its effects on the various fermion observables, can be easily obtained, as I will show with an explicit example.

One might ask the question of why should the replacement of Equation (12) be considered as the unique one. In my opinion, there exists a deep reason that motivates the procedure. In an extended treatment of the Electroweak Interactions based on the Standard Model approach, the request is advanced that the theoretical description has a symmetry under local gauge transformations of the fermion and electroweak fields. This invariance of the theory is met if the replacement Equation (12) is applied (including the new weak fields). But in order that the formalism can maintain contact with reality (providing masses to the $\mathrm{W}$ and $\mathrm{Z}$ bosons), a Higgs mechanism must be introduced, together with a proper real scalar particle: the Higgs boson. The fact that the latter one has been finally discovered at LHC seems (to me) to justify the fact that the "minimal" prescription Equation (12) is accepted to describe the electromagnetic interaction of a fermion field. To derive the modifications of the various fermion observables, i.e. the effects of the electromagnetic interaction Equation (12) on the fermion system, is now straightforward. Particularly simple is the case of the fermion orbital angular momentum $\boldsymbol{L}$. Starting from its free expression Equation (4) one simply replaces the components $\partial_{\mathrm{i}}$ of $\nabla$ by their covariant generalizations

$$
\partial_{\mathrm{i}} \rightarrow \partial_{\mathrm{i}}+\mathrm{i} e A_{\mathrm{i}}(x) .
$$

This replacement generates the new expression of the orbital angular momentum $\boldsymbol{L}^{\prime}$, that I shall write finally as:

$$
\boldsymbol{L}^{\prime}=\boldsymbol{L}+\Delta_{A} \boldsymbol{L}
$$

where the electromagnetic effect on $\boldsymbol{L}$ is simply

$$
\Delta_{A} \boldsymbol{L}=e \int_{V_{A}} \mathrm{~d}^{3} x \psi^{\dagger}(x)[\boldsymbol{x} \wedge \boldsymbol{A}(x)] \psi(x)
$$

As one sees, only the space components $A_{i}(x), i=1,2,3$, are involved in the effect. Note that the integration will now be performed only in that space volume $V_{A}$ where these components are not vanishing, which will be fixed by the proper specific choice of the electromagnetic field, to be decided by the circumstances which one wants to investigate. In this paper, the circumstance that I want to study is one in which an elementary fermion system, described by a quantum field $\psi(x)$, is suddenly hit by an intense electromagnetic field, artificially produced by some proper experimental apparatus. To describe this interaction, I will follow the ancient Dirac's treatment of this situation (see e.g. the discussion found in [9]), and treat the electromagnetic field as a classical one, retaining however the same "minimal" form (12) of the covariant derivative. This will lead to 
the same expression Equation (15) of the orbital momentum variation, where now $A_{\mu}$ is the classical vector potential, related to the classical electric and magnetic fields by the Maxwell equations. To avoid confusion, I will call this variation

$$
\Delta_{A^{c}} \boldsymbol{L}=e \int \mathrm{d}^{3} x \psi^{\dagger}(x)\left[\boldsymbol{x} \wedge \boldsymbol{A}^{c}(x)\right] \psi(x)
$$

where $\boldsymbol{A}^{c}(x)$ is a classical quantity, fixed as I said from chosen circumstances to be investigated. A particular simple and realistic case is that of a constant magnetic field $\mathcal{H}$. Fixing the $z$ axis as the field direction, we shall write

$$
\mathcal{H} \equiv(0,0, \mathcal{H})
$$

In this situation we know that

$$
\boldsymbol{A}=\frac{1}{2}(\mathcal{H} \wedge \boldsymbol{r})
$$

i.e.

$$
\boldsymbol{A}=\left(A^{1}, A^{2}, 0\right)
$$

with

$$
\begin{aligned}
A^{1} & =-\frac{1}{2} x^{2} \mathcal{H} \\
A^{2} & =\frac{1}{2} x^{1} \mathcal{H}
\end{aligned}
$$

The effects of the electromagnetic interaction on $\boldsymbol{L}$ are therefore completely provided by the magnetic field. Its explicit expressions are:

$$
\begin{aligned}
& \Delta_{A^{c}} L^{1}=-\mathcal{H}_{\frac{e}{2}} \int_{V_{\mathcal{H}}} \mathrm{d}^{3} x \psi^{\dagger}(x)\left[x^{1} x^{3}\right] \psi(x) \\
& \Delta_{A^{c}} L^{2}=-\mathcal{H}_{\frac{e}{2}} \int_{V_{\mathcal{H}}} \mathrm{d}^{3} x \psi^{\dagger}(x)\left[x^{2} x^{3}\right] \psi(x) \\
& \Delta_{A^{c}} L^{3}=\mathcal{H}_{\frac{e}{2}} \int_{V_{\mathcal{H}}} \mathrm{d}^{3} x \psi^{\dagger}(x)\left[\left(x^{1}\right)^{2}+\left(x^{2}\right)^{2}\right] \psi(x)
\end{aligned}
$$

and the integration is performed in the volume occupied by the magnetic field. This requires a more precise statement: the magnetic field is assumed to be constant (and given by Equation (17)) inside a finite volume $V_{\mathcal{H}}$, and vanishing outside. As a consequence, only the values of the fermion field inside this finite volume will be affecting the orbital angular momentum charge, as one might have imagined. Equations (22)-(24) describe the complete effect of the considered $\mathcal{H}$ field. The next relevant aim is that of computing, for a realistic elementary quantum fermion system, the mean value of these effects. Before moving to this calculation, one may stress a number of features of Equations (22)-(24) that appear to be rather general. In particular:

1. The effect is proportional to the intensity of the magnetic field, and to the electric charge $e$.
2. The effect depends strongly on the shape of the chosen volume where $H$ is different from zero.

3. The effects on the $\boldsymbol{L}$ components are given by integrals of the positive quantity $\psi^{\dagger} \psi$ multiplied by different weight functions, that can assume either positive or negative values for $L^{1,2}$, but always positive values for $L^{3}$. Thus $\Delta L^{3}$ will certainly have the sign of $\mathcal{H}$. Also, an inversion of direction of $H$ will change the sign of the effect (but not its size). This last property will be true also for the two remaining components $L^{1,2}$.

To compute the effect on the spin components is less immediate, because Equation (5) does not contain, in the integral, derivatives of the field to which the minimal procedure of Equation (12) can be applied. A possible approximated approach can be followed, and I will show it now. The starting idea is to provide a parametrization of the effect of an introduced electromagnetic field on the fermion fields $\psi(x), \bar{\psi}(x)$. With this purpose, I rewrite the Dirac equations for free $\psi$ and $\bar{\psi}$ in the following way:

$$
\begin{aligned}
& \psi(x)=\frac{1}{m}\left[\mathrm{i} \gamma^{\mu} \partial_{\mu} \psi(x)\right] \\
& \bar{\psi}(x)=-\frac{1}{m}\left[\mathrm{i}\left(\partial_{\mu} \bar{\psi}(x)\right) \gamma^{\mu}\right]
\end{aligned}
$$

Introducing an electromagnetic field will be described by the accepted "minimal" modification of Equation (12). In this way, Equations (25) and (26) will become:

$$
\begin{aligned}
& \psi(x)=\frac{1}{m}\left[\mathrm{i} \gamma^{\mu} \partial_{\mu} \psi(x)-e \gamma^{\mu} A_{\mu} \psi(x)\right] \\
& \bar{\psi}(x)=-\frac{1}{m}\left[\mathrm{i}\left(\partial_{\mu} \bar{\psi}(x)\right) \gamma^{\mu}+e \bar{\psi}(x) \gamma^{\mu} A_{\mu}\right]
\end{aligned}
$$

One can see Equations (27) and (28) as describing the shift on the fields due to the introduction of the electromagnetic field. I will write them in the following way:

$$
\begin{aligned}
& \psi(x)=\psi(x)^{(A=0)}+\Delta_{A} \psi(x) \\
& \bar{\psi}(x)=\bar{\psi}(x)^{(A=0)}+\Delta_{A} \bar{\psi}(x)
\end{aligned}
$$

where

$$
\begin{aligned}
& \Delta_{A} \psi(x)=-\frac{e}{m} \gamma^{\mu} A_{\mu} \psi(x) \\
& \Delta_{A} \bar{\psi}(x)=-\frac{e}{m} \bar{\psi}(x) \gamma^{\mu} A_{\mu} .
\end{aligned}
$$

In the following part of this section, I will be limited to a "lowest order" approximation in the calculation of the electromagnetic effects.

Given the smallness of the electric charge $e$ which appears in the interaction, I will only consider the lowest order terms in $e$ in any relevant quantity. This means that I will write Equations (31) and (32) as: 


$$
\begin{gathered}
\Delta_{A} \psi(x)=-\frac{e}{m} \gamma^{\mu} A_{\mu} \psi(x)^{(A=0)} \\
\Delta_{A} \bar{\psi}(x)=-\frac{e}{m} \bar{\psi}(x)^{(A=0)} \gamma^{\mu} A_{\mu} .
\end{gathered}
$$

I can now compute the effects on $A_{\mu}$ on the spin angular momentum replecing in (5) the fermion fields with the expressions (29), (30). In this way I would obtain four terms. One of them would correspond to the $A=0$ situation. In the next three quanities, one would be of order $e^{2}$ (coming from $\Delta_{A} \bar{\psi} \Delta_{A} \psi$ ), and I will neglect it. Therefore, to the lowest order in the electric charge $e, \mathrm{I}$ will obtain:

$$
\begin{aligned}
\Delta_{A} S_{l m} & =\frac{e}{2 m} \int \mathrm{d}^{3} x \bar{\psi}(x)^{(A=0)} \\
& \cdot\left\{\gamma^{0} \sigma^{l m} \gamma^{\mu} A_{\mu}+\gamma^{\mu} A_{\mu} \gamma^{0} \sigma^{l m}\right\} \psi(x)^{(A=0)}
\end{aligned}
$$

Equation (35) is valid for a general electromagnetic field $A_{\mu}(x)$. For the practical purposes of this paper I will now consider, as I did previously, a classical electromagnetic field by which the fermion system is supposed to be irradiated, and denote the effect of Equation (35) as $\Delta_{A^{c}} S_{l m}$. One notices at this point that, in the expression of the shift, in addition to the contribution of the space components $A^{i}(i=1,2,3)$, there appears also a new contribution from $A_{0}$, that was not present for the orbital angular momentum. To avoid this separate behaviours I will follow the allowed procedure [10] of working in a gauge where $A_{0}$ is zero. With this choice, after a number of straightforward steps that use the definitions and the properties of the $\gamma$ matrices, I am led to the final expression:

$$
\Delta_{A^{c}} S_{l m}=\frac{-\mathrm{i} e}{m} \int \mathrm{d}^{3} x\left[\psi^{\dagger}(x)^{(A=0)}\left(A_{1} \gamma^{m}-A_{m} \gamma^{l}\right)\right] \psi(x)^{(A=0)}
$$

which can be formally written as:

$$
\Delta_{A^{c}} \boldsymbol{S}=\frac{e}{m} \int \mathrm{d}^{3} x \psi^{\dagger}(x)^{(A=0)}\left[\mathrm{i} \gamma \wedge \boldsymbol{A}^{c}(x)\right] \psi(x)^{(A=0)}
$$

showing an impressive analogy with the corresponding expression for $\boldsymbol{L}$, Equation (16), with the simple correspondence $x \leftrightarrow \mathrm{i} \gamma$. In particular, we can choose again the configuration of a constant magnetic field, already fixed by Equation (17), with the direction of the $z$ axis. In this case, we shall obtain:

$$
\begin{aligned}
& \Delta_{A^{c}} S^{1}=-\mathcal{H}_{\frac{e}{2 m}} \int_{V_{\mathcal{H}}} \mathrm{d}^{3} x \psi^{\dagger}(x)^{(A=0)}\left[x^{1} \mathrm{i} \gamma^{3}\right] \psi(x)^{(A=0)} \\
& \Delta_{A^{c}} S^{2}=-\mathcal{H}_{\frac{e}{2 m}} \int_{V_{\mathcal{H}}} \mathrm{d}^{3} x \psi^{\dagger(A=0)}(x)\left[x^{2} \mathrm{i} \gamma^{3}\right] \psi^{(A=0)}(x) \\
& \Delta_{A^{c}} S^{3}=\mathcal{H}_{\frac{e}{2 m} V_{\mathcal{H}}} \mathrm{d}^{3} x \psi^{\dagger(A=0)}(x)\left[x^{1} \gamma^{1}+x^{2} \gamma^{2}\right] \psi^{(A=0)}(x) .
\end{aligned}
$$

Equations (38)-(40) exhibit a few general properties that are quite similar to those for $\Delta_{A^{c}} \boldsymbol{L}$ listed after (22)(24). In particular, the effect is again proportional to $\mathcal{H}$ and $e$, and depends strongly on the shape of $V_{\mathcal{H}}$. It should be observed that the introduction of the considered magnetic field produces automatically effects on $\boldsymbol{L}$ and $\boldsymbol{S}$ that have the same dependence on $\mathcal{H}$, so that given the form of the considered $\mathcal{H}$ one can compute immediately both. As a unique and extremely simplified example of the previous equations on a fermionic state, I have considered for pure illustration the case of the one fermion state defined by Equation (6). In fact, in the derived Equations (22)-(24) and (38)-(40) the fields $\psi, \psi^{\dagger}$ that appear in the integral must be considered, at lowest order, as free $(A=0)$ fermion fields, so that the expansions (1), (2) are still valid. The calculation of the mean values can therefore be performed as in Section 2 , and its main features can be summarized as follows. For $\boldsymbol{L}$, I derive the expressions:

$$
\begin{aligned}
\Delta_{A^{c}}\left\langle L^{1,2}\right\rangle_{\boldsymbol{k}_{0}} & =-\frac{\mathcal{H} e}{2} u^{\dagger s_{0}}\left(\boldsymbol{k}_{0}\right) u^{s_{0}}\left(\boldsymbol{k}_{0}\right) \int_{V_{\mathcal{H}}} \mathrm{d}^{3} x x^{1,2} x^{3} \\
\Delta_{A^{c}}\left\langle L^{3}\right\rangle_{\boldsymbol{k}_{0}}= & \frac{\mathcal{H} e}{2} u^{\dagger s_{0}}\left(\boldsymbol{k}_{0}\right) u^{s_{0}}\left(\boldsymbol{k}_{0}\right) \\
& \cdot \int_{V_{\mathcal{H}}} \mathrm{d}^{3} x\left[\left(x^{1}\right)^{2}+\left(x^{2}\right)^{2}\right]
\end{aligned}
$$

where, in the chosen convention [4]:

$$
u^{\dagger s_{0}}\left(\boldsymbol{k}_{0}\right) u^{s_{0}}\left(\boldsymbol{k}_{0}\right)=2 E_{\boldsymbol{k}_{0}}=2 \sqrt{\boldsymbol{k}_{0}^{2}+m^{2}} .
$$

We see that the effect will be certainly not vanishing for $L^{3}$, while for $L^{1,2}$ the effect depends strongly on the choice of $V_{\mathcal{H}}$, and might be made vanishing in principle. In the case of $S$, the main result is that for the chosen state $\Delta_{A^{c}} S^{3}$ will be always vanishing since $u^{\dagger} \gamma^{1,2} u=0$. For the remaining components we shall have to perform a more realistic calculation which goes beyond the case of a simple $\left|\boldsymbol{k}_{0}\right\rangle$ state. Note that the quantity $\psi^{\dagger}(x) \mathrm{i} \gamma^{3} \psi(x)$ is always real, as one expects, since the imaginary constant multiplies an extra imaginary term.

Although the choice of the fermion state is simply a very special illustration of the expected realistic effects, one expects that a constant magnetic field will certainly produce an effect on $L^{3}$, i.e. the quantity that was vanishing for a free fermion state, and might not affect $S^{3}$, i.e. the only spin quantity that was not vanishing for the same free state. For the remaining $\boldsymbol{L}$ and $\boldsymbol{S}$ components, a more specific calculation must be performed, which also inserts the experimentally used values of the magnetic field and the shape of this volume where it is located. This calculation, which is beyond the purposes of this preliminary paper, is now being performed in 
collaboration with a group that includes medical colleagues [11].

\section{Conclusions}

This paper has tried to show the fact that a magnetic field alone would affect two characteristic properties, i.e. the values of the orbital and spin angular momentum, of an elementary component of the existing known matter, taken to be a one electron state. The computed effects are simple and, in principle, calculable. In an extremely simplified case for the one electron system, a few properties have been found that show that the effects on the separate components of the angular momentum are essentially correlated. Also, the effects are apparently concentrated on those components of the two angular momenta that would be mostly depressed in the absence of the field. These features are reasonably general and deserve a more specific investigation in the case of a realistic fermion system.

From a certain point of view, this paper represents in my opinion a first effort to enter in communication with the fascinating ideas that medicine has very recently developed, that I tried to summarize in the Introduction. In particular, I am thinking of the effects that have been seen of a magnetic field on the elementary component of the human cells, the nucleus. These act on the two components of the nucleus, the DNA and the "epigenomic" one, in a way that is being deeply investigated at the moment, and modify both [12]. In my extremely personal view, I like to consider the electron spin, i.e. a totally intrinsic property that it has independently of the surrounding environment, as the analogue of the nucleus DNA. The electron orbital angular momentum might then correspond to the nucleus epigenoma, and the correlations of the effects of a magnetic field on the two components of the angular momentum might find an analogy with what happens in the case of the cell nucleus. This might provide a simple and useful information. Whether this personal idea (that is in fact a personal hope) might be realistically confirmed will be verified in a near future by the collaboration that I mentioned in the paper with a group of medical doctors. The results of this collaboration might appear soon and, as one old Italian statement says: "If they are roses, they will bloom".

\section{Acknowledgements}

It is a pleasure for me to thank Mario Biava and Fabio Burigana for several discussions and explanations that I had from them on essential details of the medical facts that I tried to reproduce.

\section{REFERENCES}

[1] Auditorium Wärtsilä Italia SpA, "On the Relationship between Cancer Stem Cells, Cancer and Neurodegenerative Illnesses," Bagnoli della Rosandra, Trieste, 15th February 2012.

[2] $\mathrm{AMeC}$, Association for Medicine and Complexity/Medicine and Complexity Association.

[3] Consiglio Nazionale delle Ricerche, "Non Ionizing High Frequency EM Radiation: Researching the Epidemiological and Chemical Evidences," First International Medical Scientific Congress, Rome, 29-30 November 1999.

[4] P. Girdinio, "An Introduction to Electromagnetic Fields and Their Biological Effects," First International Medical Scientific Congress, Rome, 29-30 November 1999, p. 133.

[5] C. Ventura, et al., FASEB Journal, Vol. 19, 2005, p. 155.

[6] C. Ventura, et al., Cell Transplant, Vol. 6, 2012, p. 1225.

[7] M. E. Peskin and D. V. Schroeder, "An Introduction to quantum field theory," Addison-Wesley, Reading, 1995.

[8] E. Noether, Gott. Nachr., Vol. 1918, 1918, pp. 235-257.

[9] A. Messiah, "Quantum Mechanics,” Dover, 1999.

[10] N. N. Bogoliukov and D. V. Shirkov, "Introduction to the Theory of Quantized Fields," Interscience Publishers, 1959.

[11] A. Bassi, M. Biava, F. Burigana and C. Verzegnassi, Work in Preparation.

[12] M. Biava, Private Communication. 